\title{
Covid19 is nothing but Lemierre's syndrome - the "forgotten disease".
}

\author{
Sandeep Chakraborty
}

\section{Letter}

I will just quote Wikipedia https://en.wikipedia.org/wiki/Lemierre\%27s_syndrome. My words are in italics, everything else is Wikipedia.

The bacteria causing the thrombophlebitis are anaerobic bacteria that are typically normal components of the microorganisms that inhabit the mouth and throat. Species of Fusobacterium, specifically Fusobacterium necrophorum, are most commonly the causative bacteria, but various bacteria have been implicated. F. necrophorum produces hemagglutinin which causes platelet aggregation that can lead to diffuse intravascular coagulation and thrombocytopenia.

Remember this paper - "The usual anaerobic bacterial suspects extracted from a global metagenomic database of Covid19 patients from Peru, Cambodia, China, Brazil and the US - Prevotella, Veillonella, Capnocytophaga, Fusobacterium, Oribacterium and Bacteroides should be monitored for colonization"

F. necrophorum produces hemagglutinin, so does Prevotella. Note, the connection to Spanish Flu - H1N1 also has hemagglutinin. So, only this time SARS-Cov2 is probably enabling Prevotella more, whatever be the reason. And this starts not in the peritonsillar region, but somewhere in the lungs - the abscess being enabled by the virus

\section{Mechanism:}

1. Lemierre's syndrome occurs most often when a bacterial (e.g., Fusobacterium necrophorum) throat infection progresses to the formation of a peritonsillar abscess. Deep in the abscess, anaerobic bacteria can flourish.

2. During the primary infection, F. necrophorum colonizes the infection site and the infection spreads to the parapharyngeal space.

3. The bacteria then invade the peritonsillar blood vessels where they can spread to the internal jugular vein.

4. In this vein, the bacteria cause the formation of a thrombus containing these bacteria.

5. In the lungs, the bacteria cause abscesses, nodulary and cavitary lesions. Pleural effusion is often present

6. Production of bacterial toxins such as lipopolysaccharide leads to secretion of cytokines by white blood cells which then both lead to symptoms of sepsis

7. signs of a bacterial infection with elevated C-reactive protein, erythrocyte sedimentation rate and white blood cells (notably neutrophils).

\section{Symptoms:}

1. usually start with a sore throat, fever, and general body weakness.

2. extreme lethargy, spiked fevers, rigors, swollen cervical lymph nodes, and a swollen, tender or painful neck.

3. Often there is abdominal pain, diarrhea, nausea and vomiting during this phase.

4. shortness of breath, cough and painful breathing (pleuritic chest pain). 


\section{Treatments:}

1. Lemierre's syndrome is primarily treated with antibiotics given intravenously. Fusobacterium necrophorum is generally highly susceptible to beta-lactam antibiotics, metronidazole, clindamycin and third generation cephalosporins while the other fusobacteria have varying degrees of resistance to betalactams and clindamycin.

\section{References}

